\title{
The stream fish fauna from the rio Machado basin, Rondônia State, Brazil
}

\author{
Lilian Casatti ${ }^{1 *}$, María Angélica Pérez-Mayorga ${ }^{1}$, Fernando Rogério Carvalho ${ }^{1}$, Gabriel Lourenço \\ Brejão ${ }^{1}$ and Igor David da Costa ${ }^{2}$ \\ 1 Universidade Estadual Paulista “Júlio de Mesquita Filho", Departamento de Zoologia e Botânica, Laboratório de Ictiologia. Rua Cristóvão Colombo, \\ 2265. CEP 15054-000. São José do Rio Preto, SP, Brazil. \\ 2 Universidade Federal de Rondônia, Departamento de Engenharia de Pesca e Aquicultura, Rua da Paz, 4376. CEP 76916-000. Presidente Médici, \\ RO, Brazil. \\ * Corresponding author: E-mail: licasatti@gmail.com
}

\begin{abstract}
The rio Machado (also known as Ji-Paraná) is a tributary of the rio Madeira in the Amazon basin. Currently, the rio Madeira contains the greatest fish species richness of the world, with approximately 1,000 species. The present study presents the fish inventory from streams of the rio Machado basin. In total, 75 stream reaches, 80 meter-length, randomly selected, were sampled in 2011 (August to October) and 2012 (June to July). Overall, 22,875 fish in eight orders, 32 families, 89 genera, and 140 species were collected. Richness estimators indicate that almost 90\% of the expected richness was registered. The great majority of specimens (52.2\%) was represented by small sized piabas such as Serrapinnus aff. notomelas, Moenkhausia collettii, Serrapinnus microdon, and Hemigrammus melanochrous. Of the total richness, 25 species were restricted to $9^{\circ} 00^{\prime} \mathrm{S}$ and $10^{\circ} 00^{\prime} \mathrm{S}$; among them, 14 were exclusive to the lower portion of the basin, which exhibits the larger proportion of native vegetation covering.
\end{abstract}

\section{INTRODUCTION}

In the Brazilian Amazon, the Rondônia State has been widely exposed to the effects resulting from deforestation. In 2001, 50.9\% of the total area had been cleared; in 2004, this percentage increased to 57.1\%; and in 2006, this percentage increased to $65.9 \%$ (INPE 2010). According to Dale et al. (1993), during 70's and 80's, the deforestation rate in the state of Rondônia has increased at a faster rate than anywhere else in the world. The highest level of deforestation occurs in the rio Machado basin, which drains the most populated area of the state because of its proximity to highway BR-364 (Fernandes and Guimarães 2002); within this basin, the central portion is the most deforested due to the occurrence of eutrophic soils (Krusche et al. 2005). Along the rio Machado basin, many upland streams have intermittent dry stretches in the dry season, and this situation has become more common recently because of the complete deforestation of many headwaters (Fernandes and Guimarães 2002).

Neotropical streams are very special ecosystems because they have many endemic species and are dominated by small-size species that generally correspond to approximately $50 \%$ of the known fish diversity (Castro et al. 2003). Proportional to the water volume available, streams are environments with high richness and, as noted by Castro and Menezes (1998) approximately 15 years ago, the study of systematics, evolution and the general biology of small fish species is undoubtedly the greatest challenge of Neotropical ichthyology. This knowledge starts with inventory studies, which are essential to manage and preserve an area or ecosystem due to the basic information provided. This type of study is even more urgent and necessary in situations in which there is a high threat of habitat loss, such as in the rio Machado basin. Therefore, our aim was to present the results of an inventory conducted in the streams of the rio Machado basin, with a species estimation analysis and an analysis of the latitudinal species distribution.

\section{MATERIAls AND Methods}

Study area and site selection

The rio Machado basin, formed by the confluence of the Comemoração and Pimenta Bueno rivers (Figure 1), has $75,400 \mathrm{~km}^{2}$. The rio Machado is approximately $1,200 \mathrm{~km}$ long and receives five other tributaries (Rolim de Moura, Urupá, Jaru, Machadinho, and Preto rivers), flowing on the right bank of the rio Madeira (Krusche et al. 2005). Its flood regime, obtained from the data set for a five-year (2008-2012) period monthly average, is characterized by rising water between November and December, high water between January and March, with the highest water level in February; the falling water period is between April and July, the low water period between August and October with the minimum water level in September (ANA 2013). The basin has an average slope of 0.62 degrees. The climate is humid tropical, with temperatures from $19^{\circ} \mathrm{C}$ to $33^{\circ} \mathrm{C}$ and annual rainfall of $2,500 \mathrm{~mm}$ (Krusche et al. 2005). The land cover of the region includes primary forest (open humid tropical forest), secondary forest, and pasture (Ferraz et al. 2009).

The sampling design, local variable assessment, and fish collections were conducted during 2011 and 2012. The watershed limits were generated with the hydrological model ArcSWAT and digital elevation models (DEM) SRTM (90 x 90 m resolution) produced by the National Aeronautics and Space Administration (NASA) and are available from the United States Geological Survey (USGS). All the selected microbasins had a minimum contribution area between 1,500 and 5,000 ha. 


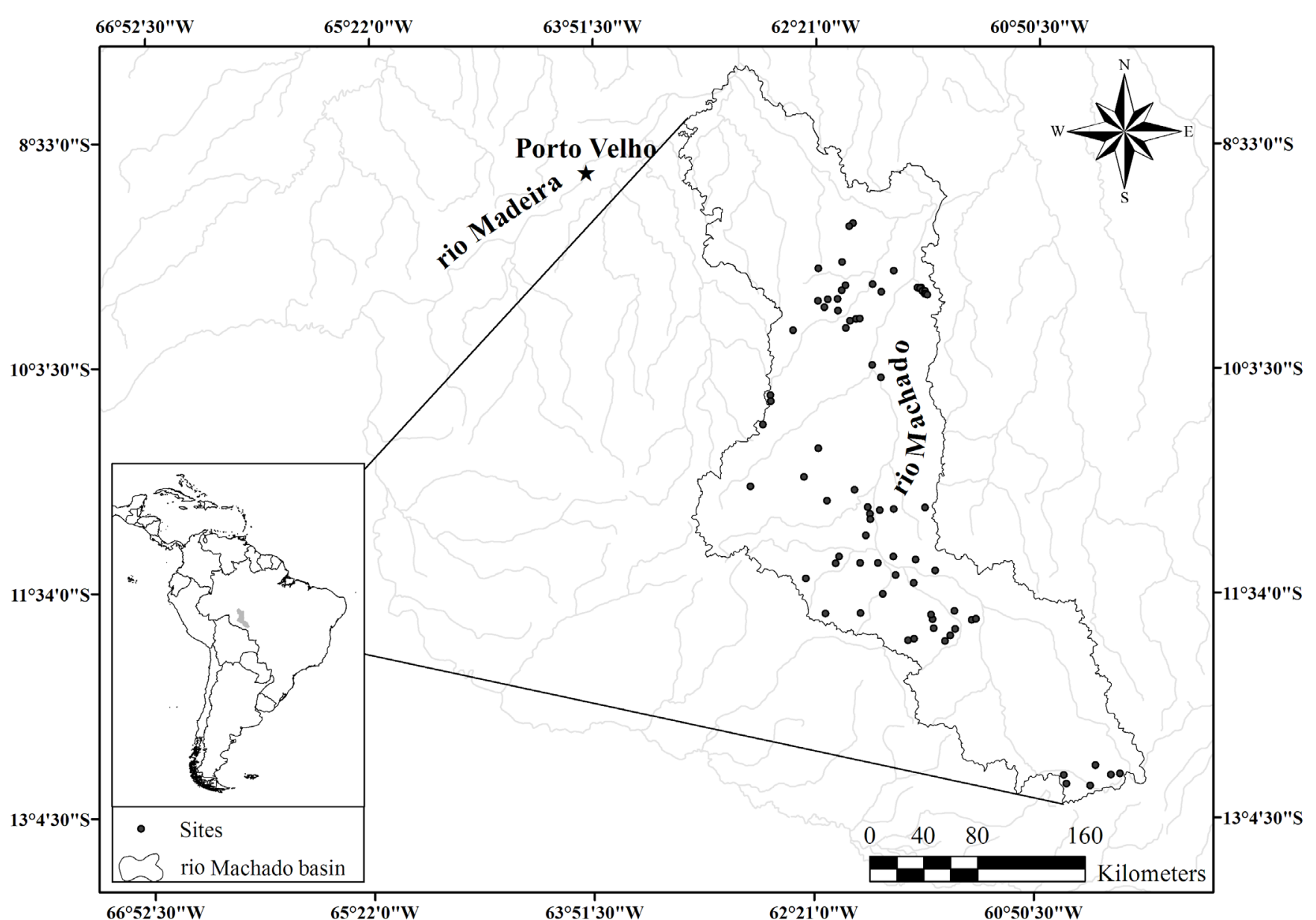

FIGURE 1. Location of the rio Machado basin in South America (small box on the left) and 75 sampling reaches in the rio Machado, Rondônia State, Brazil (black dots on the right). Some symbols are superimposed due to the proximity of sites.

\section{Fish collection and identification}

A total of 75 reaches (Table 1) were sampled once during the dry season (August to October, 2011 and June to July, 2012). Each headwater stream reach $\left(1^{\text {st }}\right.$ to $3^{\text {rd }}$ order reaches according to Sthraler 1957) was $80 \mathrm{~m}$ long and was sampled after blocking the reaches up- and downstream using block nets (5 $\mathrm{mm}$ mesh). During one hour, two collectors sampled fish with a seine $(1.5 \times 2 \mathrm{~m}$, $2 \mathrm{~mm}$ mesh $)$ and a dip net $(0.5 \times 0.8 \mathrm{~m}, 2 \mathrm{~mm}$ mesh $)$. Fish were collected under ICMBio (Instituto Chico Mendes de Conservação da Biodiversidade) permits (4355-1/2012). The fish identification was conducted by consulting specialists, and voucher specimens are deposited in the Coleção de Peixes do Departamento de Zoologia e Botânica (DZSJRP) at the Universidade Estadual Paulista "Júlio de Mesquita Filho", São José do Rio Preto, São Paulo State, Brazil (Table 2).

\section{Data analysis}

To evaluate the inventory representativeness, the Coleman rarefaction (Colwell et al. 2004) was obtained and compared to two non parametric richness estimators, the ICE (Incidence Coverage Estimator, Lee and Chao 1994) and the ACE (Abundance Coverage Estimator, Lee and Chao 1994), using the software EstimateS 7.5.2 (Cowell 2005). The latitudinal distribution of the fish fauna was evaluated by calculating the average and extreme values of latitude, considering all stream reaches in which each species occurs.

\section{RESULTS AND DiscusSion}

A total of 22,875 specimens belonging to 140 species, 89 genera, 32 families, and eight orders were collected (Table 2). The Characiformes and Siluriformes, representing 51 and $31 \%$ of the total species, respectively, were predominant, which is consistent with the prevalence previously noted for the streams and rivers of the Neotropical region (Lowe-McConnell 1999). The families with the highest abundance in the sampled reaches were Characidae, Cichlidae, and Loricariidae, which are taxa that are broadly distributed in the Amazon basin (Reis et al. 2003). Aequidens tetramerus, Bryconops caudomaculatus, Characidium aff. zebra, Creagrutus petilus, Crenicichla santosi, Moenkhausia oligolepis, M. collettii, Phenacogaster retropinnus, and Rineloricaria heteroptera were broadly distributed in the rio Machado basin; they were recorded in more than $50 \%$ of the reaches. In contrast, 26 species (18.6\%) occurred only in one site; most of them were recorded from the lower portion of the basin. Non-native species were represented by two specimens of Tilapia rendalli, and currently, the non-native threats appear to be of minor concern in the rio Machado basin. However, because of the recent development of aquaculture in the basin (author's observation), it is critical that appropriate precautions are taken so that non-native species do not become a threat to the local fish fauna.

The largest percentage of the fish abundance (52.2\%) was represented by small-sized "piabas" of the family Characidae, such as Serrapinnus aff. 
notomelas, Moenkhausia collettii, Serrapinnus microdon, Hemigrammus melanochrous, Hyphessobrycon agulha, Creagrutus petilus, and Bryconops caudomaculatus, in this order. The predominance of small fish species is in agreement with the overall pattern for South American stream fish and was explained by Castro (1999) as a result of selective pressures of the lotic environment in addition to the combination of geological history of South American basins, vicariant processes and allopatric speciation. Another common pattern is the great number of rare species. Of the total richness, 53 species (37.9\%) were represented by less than ten specimens, and 14 species $(10 \%)$ were represented by a single specimen, such as Corydoras bondi and Miuroglanis platycephalus, which indeed are species represented in fish collections by only a few records $(\leq 10)$ (SpeciesLink 2013).

The richness estimated with ACE and ICE was 148 and 157 species, respectively, which indicates that more than
$90 \%$ of the estimated species richness was registered and shows good representativeness of the inventory (Figure 2). Inventories of ichthyofauna for the western Amazon region are scarce. Among those that have been conducted in the rio Madeira basin, we highlight the study by Perin et al. (2007), which recorded 48 fish species in an urban area of Rondônia; the study by Camargo and Giarrizzo (2007), which recorded 133 species in 23 streams and three rivers of the Marmelos Preservation Area; and the study by Barros et al. (2011), which recorded 78 species in 22 streams in the Madeira-Purus interfluvial region. Despite the different sampling methodologies employed among these studies, they clearly demonstrate the great fish diversity in the rio Madeira basin, with a high percentage of species yet to be found and described.

Of the total species collected, 97 (69.3\%) were identified to species level, $43(30.7 \%)$ are of uncertain taxonomic status, because they are not formally described ( 16 species)

FIGURE 1. Municipality, altitude (m), and geographical coordinates of the 75 stream reaches sampled in the rio Machado basin. Sites 24 to 31 are located in the Reserva Biológica (REBio) Jaru, 33 to 35 in the Reserva Extrativista (RESEX) Rio Preto-Jacundá, 38 and 39 in the RESEX Castanheira, and 43 to 45 in the RESEX Aquariquara.

\begin{tabular}{|c|c|c|c|}
\hline SITES & MUNICIPALITY & ELEVATION & $\begin{array}{l}\text { GEOGRAPHICAL } \\
\text { COORDINATES }\end{array}$ \\
\hline 1 & Presidente Médici & 196.64 & $62^{\circ} 00^{\prime} 21^{\prime \prime} \mathrm{W}, 11^{\circ} 12^{\prime} 06^{\prime \prime} \mathrm{S}$ \\
\hline 2 & Alvorada d'Oeste & 251.97 & $62^{\circ} 24^{\prime} 55^{\prime \prime} \mathrm{W}, 11^{\circ} 29^{\prime} 31^{\prime \prime} \mathrm{S}$ \\
\hline 3 & Teixerópolis & 193.24 & $62^{\circ} 16^{\prime} 18^{\prime \prime} \mathrm{W}, 10^{\circ} 58^{\prime} 06^{\prime \prime} \mathrm{S}$ \\
\hline 4 & Ji-Paraná & 183.93 & $62^{\circ} 05^{\prime} 03^{\prime \prime} \mathrm{W}, 10^{\circ} 53^{\prime} 45^{\prime \prime} \mathrm{S}$ \\
\hline 5 & Nova União & 198.81 & $62^{\circ} 47^{\prime} 28^{\prime \prime} \mathrm{W}, 10^{\circ} 52^{\prime} 20^{\prime \prime} \mathrm{S}$ \\
\hline 6 & Nova União & 199.44 & $62^{\circ} 25^{\prime} 38^{\prime \prime} \mathrm{W}, 10^{\circ} 48^{\prime} 38^{\prime \prime} \mathrm{S}$ \\
\hline 7 & Ouro Preto d'Oeste & 226.68 & $62^{\circ} 19^{\prime} 56^{\prime \prime} \mathrm{W}, 10^{\circ} 36^{\prime} 52^{\prime \prime} \mathrm{S}$ \\
\hline 8 & Castanheiras & 190.74 & $61^{\circ} 55^{\prime} 23^{\prime \prime} \mathrm{W}, 11^{\circ} 23^{\prime} 15^{\prime \prime} \mathrm{S}$ \\
\hline 9 & $\begin{array}{l}\text { Nova Brasilândia } \\
\text { d'Oeste }\end{array}$ & 286.23 & $62^{\circ} 16^{\prime} 48^{\prime \prime} \mathrm{W}, 11^{\circ} 43^{\prime} 38^{\prime \prime} \mathrm{S}$ \\
\hline 10 & Rolim de Moura & 236.09 & $62^{\circ} 02^{\prime} 26^{\prime \prime} \mathrm{W}, 11^{\circ} 43^{\prime} 27^{\prime \prime} \mathrm{S}$ \\
\hline 11 & Santa Luzia d'Oeste & 254.74 & $61^{\circ} 40^{\prime} 24^{\prime \prime} \mathrm{W}, 11^{\circ} 53^{\prime} 45^{\prime \prime} \mathrm{S}$ \\
\hline 12 & Santa Luzia d'Oeste & 248.89 & $61^{\circ} 42^{\prime} 53^{\prime \prime} \mathrm{W}, 11^{\circ} 54^{\prime} 21^{\prime \prime} \mathrm{S}$ \\
\hline 13 & Presidente Médici & 180.28 & $61^{\circ} 54^{\prime} 42^{\prime \prime} \mathrm{W}, 11^{\circ} 01^{\prime} 51^{\prime \prime} \mathrm{S}$ \\
\hline 14 & Cujubim & 178.86 & $62^{\circ} 20^{\prime} 05^{\prime \prime} \mathrm{W}, 09^{\circ} 24^{\prime} 21^{\prime \prime} \mathrm{S}$ \\
\hline 15 & Machadinho d'Oeste & 184.77 & $62^{\circ} 10^{\prime} 22^{\prime \prime} \mathrm{W}, 09^{\circ} 21^{\prime} 47^{\prime \prime} \mathrm{S}$ \\
\hline 16 & São Felix & 143.06 & $61^{\circ} 49^{\prime} 19^{\prime \prime} \mathrm{W}, 09^{\circ} 25^{\prime} 17^{\prime \prime} \mathrm{S}$ \\
\hline 17 & Vale do Anari & 174.88 & $62^{\circ} 12^{\prime} 09^{\prime \prime} \mathrm{W}, 09^{\circ} 36^{\prime} 42^{\prime \prime} \mathrm{S}$ \\
\hline 18 & Vale do Anari & 198.53 & $62^{\circ} 16^{\prime} 08^{\prime \prime} \mathrm{W}, 09^{\circ} 36^{\prime} 58^{\prime \prime} \mathrm{S}$ \\
\hline 19 & Vale do Anari & 192.34 & $62^{\circ} 20^{\prime} 13^{\prime \prime} \mathrm{W}, 09^{\circ} 37^{\prime} 28^{\prime \prime} \mathrm{S}$ \\
\hline 20 & Vale do Anari & 182.19 & $62^{\circ} 17^{\prime} 29^{\prime \prime} \mathrm{W}, 09^{\circ} 40^{\prime} 06^{\prime \prime} \mathrm{S}$ \\
\hline 21 & Vale do Anari & 175.20 & $62^{\circ} 30^{\prime} 11^{\prime \prime} \mathrm{W}, 09^{\circ} 49^{\prime} 26^{\prime \prime} \mathrm{S}$ \\
\hline 22 & Vale do Anari & 119.48 & $61^{\circ} 58^{\prime} 09^{\prime \prime} \mathrm{W}, 10^{\circ} 04^{\prime} 14^{\prime \prime} \mathrm{S}$ \\
\hline 23 & Theobroma & 119.48 & $61^{\circ} 54^{\prime} 32^{\prime \prime} \mathrm{W}, 10^{\circ} 08^{\prime} 35^{\prime \prime} \mathrm{S}$ \\
\hline 24 & Vale do Anari & 103.87 & $61^{\circ} 39^{\prime} 15^{\prime \prime} \mathrm{W}, 09^{\circ} 32^{\prime} 10^{\prime \prime} \mathrm{S}$ \\
\hline 25 & Vale do Anari & 151.50 & $61^{\circ} 38^{\prime} 53^{\prime \prime} \mathrm{W}, 09^{\circ} 32^{\prime} 37^{\prime \prime} \mathrm{S}$ \\
\hline 26 & Vale do Anari & 126.54 & $61^{\circ} 38^{\prime} 40^{\prime \prime} \mathrm{W}, 09^{\circ} 32^{\prime} 31^{\prime \prime} \mathrm{S}$ \\
\hline 27 & Vale do Anari & 107.91 & $61^{\circ} 37^{\prime} 42^{\prime \prime} \mathrm{W}, 09^{\circ} 32^{\prime} 54^{\prime \prime} \mathrm{S}$ \\
\hline 28 & Vale do Anari & 103.91 & $61^{\circ} 36^{\prime} 39^{\prime \prime} \mathrm{W}, 09^{\circ} 33^{\prime} 25^{\prime \prime} \mathrm{S}$ \\
\hline 29 & Vale do Anari & 126.98 & $61^{\circ} 36^{\prime} 50^{\prime \prime} \mathrm{W}, 09^{\circ} 34^{\prime} 20^{\prime \prime} \mathrm{S}$ \\
\hline 30 & Vale do Anari & 115.46 & $61^{\circ} 36^{\prime} 26^{\prime \prime} \mathrm{W}, 09^{\circ} 34^{\prime} 43^{\prime \prime} \mathrm{S}$ \\
\hline 31 & Vale do Anari & 155.00 & $61^{\circ} 36^{\prime} 17^{\prime \prime} \mathrm{W}, 09^{\circ} 35^{\prime} 42^{\prime \prime} \mathrm{S}$ \\
\hline 32 & Machadinho d'Oeste & 192.98 & $62^{\circ} 09^{\prime} 44^{\prime \prime} \mathrm{W}, 09^{\circ} 40^{\prime} 03^{\prime \prime} \mathrm{S}$ \\
\hline 33 & Machadinho d'Oeste & 189.14 & $62^{\circ} 05^{\prime} 55^{\prime \prime} \mathrm{W}, 09^{\circ} 06^{\prime} 15^{\prime \prime} \mathrm{S}$ \\
\hline 34 & Machadinho d'Oeste & 163.65 & $62^{\circ} 07^{\prime} 28^{\prime \prime} \mathrm{W}, 09^{\circ} 07^{\prime} 17^{\prime \prime} \mathrm{S}$ \\
\hline 35 & Machadinho d'Oeste & 158.76 & $62^{\circ} 07^{\prime} 33^{\prime \prime} \mathrm{W}, 09^{\circ} 07^{\prime} 20^{\prime \prime} \mathrm{S}$ \\
\hline 36 & Machadinho d'Oeste & 129.13 & $61^{\circ} 57^{\prime} 55^{\prime \prime} \mathrm{W}, 09^{\circ} 30^{\prime} 52^{\prime \prime} \mathrm{S}$ \\
\hline 37 & Machadinho d'Oeste & 218.32 & $61^{\circ} 55^{\prime} 45^{\prime \prime} \mathrm{W}, 09^{\circ} 33^{\prime} 19^{\prime \prime} \mathrm{S}$ \\
\hline 38 & Machadinho d'Oeste & 186.78 & $62^{\circ} 10^{\prime} 30^{\prime \prime} \mathrm{W}, 09^{\circ} 33^{\prime} 15^{\prime \prime} \mathrm{S}$ \\
\hline 39 & Machadinho d'Oeste & 188.66 & $62^{\circ} 08^{\prime} 49^{\prime \prime} \mathrm{W}, 09^{\circ} 31^{\prime} 21^{\prime \prime} \mathrm{S}$ \\
\hline
\end{tabular}

\begin{tabular}{|c|c|c|c|}
\hline SITES & MUNICIPALITY & ELEVATION & $\begin{array}{l}\text { GEOGRAPHICAL } \\
\text { COORDINATES }\end{array}$ \\
\hline 40 & Ariquemes & 198.49 & $62^{\circ} 39^{\prime} 13^{\prime \prime} \mathrm{W}, 10^{\circ} 15^{\prime} 28^{\prime \prime} \mathrm{S}$ \\
\hline 41 & Theobroma & 202.45 & $62^{\circ} 38^{\prime} 40^{\prime \prime} \mathrm{W}, 10^{\circ} 18^{\prime} 12^{\prime \prime} \mathrm{S}$ \\
\hline 42 & Jaru & 199.19 & $62^{\circ} 42^{\prime} 33^{\prime \prime} \mathrm{W}, 10^{\circ} 27^{\prime} 24^{\prime \prime} \mathrm{S}$ \\
\hline 43 & Vale do Anari & 182.46 & $62^{\circ} 07^{\prime} 01^{\prime \prime} \mathrm{W}, 09^{\circ} 45^{\prime} 33^{\prime \prime} \mathrm{S}$ \\
\hline 44 & Vale do Anari & 199.70 & $62^{\circ} 05^{\prime} 58^{\prime \prime} \mathrm{W}, 09^{\circ} 45^{\prime} 00^{\prime \prime} \mathrm{S}$ \\
\hline 45 & Vale do Anari & 192.08 & $62^{\circ} 03^{\prime} 10^{\prime \prime} \mathrm{W}, 09^{\circ} 44^{\prime} 35^{\prime \prime} \mathrm{S}$ \\
\hline 46 & Vale do Anari & 194.09 & $62^{\circ} 08^{\prime} 53^{\prime \prime} \mathrm{W}, 09^{\circ} 48^{\prime} 24^{\prime \prime} \mathrm{S}$ \\
\hline 47 & Ministro Adreazza & 298.80 & $61^{\circ} 36^{\prime} 15^{\prime \prime} \mathrm{W}, 11^{\circ} 00^{\prime} 55^{\prime \prime} \mathrm{S}$ \\
\hline 48 & Presidente Médici & 210.56 & $61^{\circ} 50^{\prime} 10^{\prime \prime} \mathrm{W}, 11^{\circ} 00^{\prime} 10^{\prime \prime} \mathrm{S}$ \\
\hline 49 & $\begin{array}{l}\text { Nova Brasilândia } \\
\text { d'Oeste }\end{array}$ & 200.01 & $62^{\circ} 03^{\prime} 19^{\prime \prime} \mathrm{W}, 11^{\circ} 21^{\prime} 51^{\prime \prime} \mathrm{S}$ \\
\hline 50 & Presidente Médici & 195.01 & $61^{\circ} 49^{\prime} 04^{\prime \prime} \mathrm{W}, 11^{\circ} 20^{\prime} 14^{\prime \prime} \mathrm{S}$ \\
\hline 51 & Cacoal & 200.37 & $61^{\circ} 39^{\prime} 51^{\prime \prime} \mathrm{W}, 11^{\circ} 21^{\prime} 59^{\prime \prime} \mathrm{S}$ \\
\hline 52 & Ji-Paraná & 188.47 & $61^{\circ} 59^{\prime} 34^{\prime \prime} \mathrm{W}, 11^{\circ} 00^{\prime} 54^{\prime \prime} \mathrm{S}$ \\
\hline 53 & Ji-Paraná & 184.21 & $61^{\circ} 58^{\prime} 46^{\prime \prime} \mathrm{W}, 11^{\circ} 03^{\prime} 27^{\prime \prime} \mathrm{S}$ \\
\hline 54 & Ji-Paraná & 185.33 & $61^{\circ} 58^{\prime} 39^{\prime \prime} \mathrm{W}, 11^{\circ} 05^{\prime} 31^{\prime \prime} \mathrm{S}$ \\
\hline 55 & Alvorada d'Oeste & 200.42 & $62^{\circ} 11^{\prime} 05^{\prime \prime} \mathrm{W}, 11^{\circ} 20^{\prime} 23^{\prime \prime} \mathrm{S}$ \\
\hline 56 & Alvorada d'Oeste & 204.94 & $62^{\circ} 12^{\prime} 49^{\prime \prime} \mathrm{W}, 11^{\circ} 23^{\prime} 24^{\prime \prime} \mathrm{S}$ \\
\hline 57 & Cacoal & 191.29 & $61^{\circ} 32^{\prime} 04^{\prime \prime} \mathrm{W}, 11^{\circ} 25^{\prime} 47^{\prime \prime} \mathrm{S}$ \\
\hline 58 & Cacoal & 198.01 & $61^{\circ} 40^{\prime} 41^{\prime \prime} \mathrm{W}, 11^{\circ} 31^{\prime} 09^{\prime \prime} \mathrm{S}$ \\
\hline 59 & Castanheiras & 198.10 & $61^{\circ} 47^{\prime} 50^{\prime \prime} \mathrm{W}, 11^{\circ} 28^{\prime} 10^{\prime \prime} \mathrm{S}$ \\
\hline 60 & Castanheiras & 201.51 & $61^{\circ} 52^{\prime} 20^{\prime \prime} \mathrm{W}, 11^{\circ} 35^{\prime} 36^{\prime \prime} \mathrm{S}$ \\
\hline 61 & Vilhena & 333.78 & $60^{\circ} 27^{\prime} 46^{\prime \prime} \mathrm{W}, 12^{\circ} 52^{\prime} 24^{\prime \prime} \mathrm{S}$ \\
\hline 62 & Vilhena & 518.15 & $60^{\circ} 18^{\prime} 50^{\prime \prime} \mathrm{W}, 12^{\circ} 47^{\prime} 36^{\prime \prime} \mathrm{S}$ \\
\hline 63 & Vilhena & 565.15 & $60^{\circ} 15^{\prime} 12^{\prime \prime} \mathrm{W}, 12^{\circ} 47^{\prime} 48^{\prime \prime} \mathrm{S}$ \\
\hline 64 & Vilhena & 507.51 & $60^{\circ} 24^{\prime} 33^{\prime \prime} \mathrm{W}, 12^{\circ} 47^{\prime} 08^{\prime \prime} \mathrm{S}$ \\
\hline 65 & Chupinguaia & 375.68 & $60^{\circ} 39^{\prime} 14^{\prime \prime} \mathrm{W}, 12^{\circ} 48^{\prime} 39^{\prime \prime} \mathrm{S}$ \\
\hline 66 & Chupinguaia & 410.26 & $60^{\circ} 37^{\prime} 58^{\prime \prime} \mathrm{W}, 12^{\circ} 51^{\prime} 05^{\prime \prime} \mathrm{S}$ \\
\hline 67 & $\begin{array}{l}\text { Primavera de } \\
\text { Rondônia }\end{array}$ & 201.15 & $61^{\circ} 16^{\prime} 29^{\prime \prime} \mathrm{W}, 11^{\circ} 45^{\prime} 45^{\prime \prime} \mathrm{S}$ \\
\hline 68 & $\begin{array}{l}\text { Primavera de } \\
\text { Rondônia }\end{array}$ & 206.21 & $61^{\circ} 15^{\prime} 55^{\prime \prime} \mathrm{W}, 11^{\circ} 46^{\prime} 20^{\prime \prime} \mathrm{S}$ \\
\hline 69 & $\begin{array}{l}\text { Primavera de } \\
\text { Rondônia }\end{array}$ & 210.23 & $61^{\circ} 23^{\prime} 19^{\prime \prime} \mathrm{W}, 11^{\circ} 44^{\prime} 04^{\prime \prime} \mathrm{S}$ \\
\hline 70 & São Felipe d'Oeste & 236.71 & $61^{\circ} 24^{\prime} 10^{\prime \prime} \mathrm{W}, 11^{\circ} 49^{\prime} 53^{\prime \prime} \mathrm{S}$ \\
\hline 71 & Rolim de Moura & 296.40 & $61^{\circ} 33^{\prime} 41^{\prime \prime} \mathrm{W}, 11^{\circ} 50^{\prime} 17^{\prime \prime S}$ \\
\hline 72 & Rolim de Moura & 222.27 & $61^{\circ} 32^{\prime} 55^{\prime \prime} \mathrm{W}, 11^{\circ} 45^{\prime} 47^{\prime \prime} \mathrm{S}$ \\
\hline 73 & Rolim de Moura & 213.46 & $61^{\circ} 32^{\prime} 50^{\prime \prime} \mathrm{W}, 11^{\circ} 44^{\prime} 44^{\prime \prime} \mathrm{S}$ \\
\hline 74 & São Felipe d'Oeste & 288.21 & $61^{\circ} 28^{\prime} 07^{\prime \prime} \mathrm{W}, 11^{\circ} 56^{\prime} 08^{\prime \prime} \mathrm{S}$ \\
\hline 75 & São Felipe d'Oeste & 258.46 & $61^{\circ} 25^{\prime} 36^{\prime \prime} \mathrm{W}, 11^{\circ} 52^{\prime} 13^{\prime \prime} \mathrm{S}$ \\
\hline
\end{tabular}


or belong to genera that need more detailed review (27 species), such as Brachyhypopomus, Cetopsorhamdia, and Moenkhausia. The percentage of species with uncertain taxonomic situations is high compared with the South and Southeast regions of Brazil, but it is small when compared to other Amazonian ecoregions. Advances in the knowledge on the fish fauna from the rio Madeira basin result from recent inventories that were led by the ichthyology team at the Federal University of Rondônia (UNIR). The rio Madeira basin, among all tributaries of the Amazon basin and even when compared with another rivers in the world, has the richest freshwater fish fauna of the world, in which approximately 1,000 freshwater fish species have been recognized (W. Ohara, pers. comm.). However, in Rondônia State, accelerated deforestation is the main source of degradation to the streams, making it urgent to acquire the taxonomic, geographical, and ecological knowledge of the ichthyofauna in these environments. As a potential additional threat to this fish fauna we can cite the expansion of hydroelectric power plants.

According to the species latitudinal distribution analysis, 25 species (18\%) only occur between $9^{\circ} 00^{\prime}$ S and $10^{\circ} 00^{\prime} \mathrm{S}$ (Figure 3); among them, nine were exclusively registered in streams reaches inside protected areas (REBio Jaru, RESEX Rio Preto-Jacundá, RESEX Castanheira, and RESEX Aquariquara). Extractive reserves of Rondônia, such as the RESEX Rio Preto-Jacundá, have adopted forest

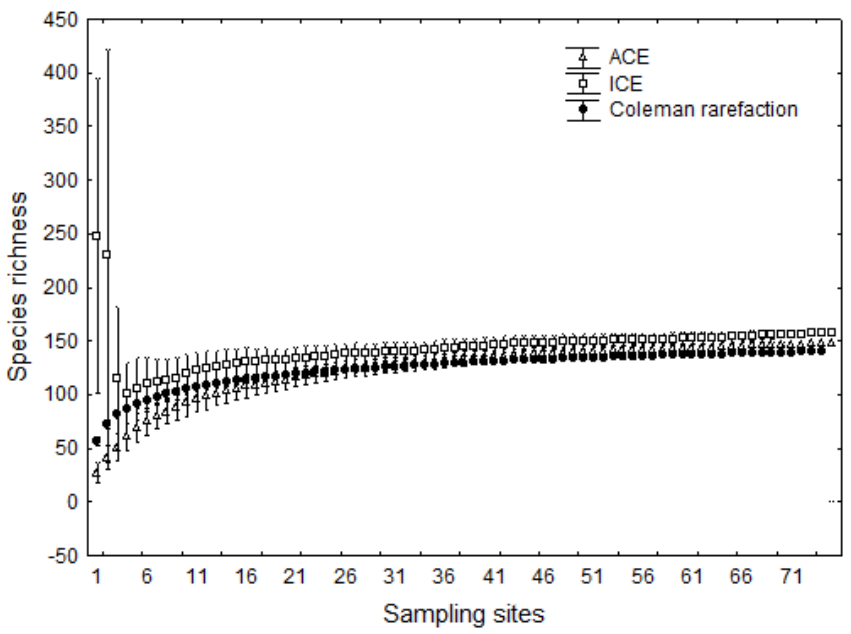

FIGURE 2. Coleman rarefaction curve, showing the observed richness, and curves of estimated number of species derived from ICE (Incidence Coverage Estimator) and ACE (Abundance Coverage Estimator) by 50 randomizations against cumulative samples.

management as an activity that is capable of generating a better quality of life for the extractive region while preserving the forest. However, as described by Martins (2008) and Moreira et al. (2010), the current forest management is not able to secure these goals, which represents a potential threat to the conservation of the regional species pool, endangering one of the world's most diverse ichthyofauna.

TABLE 2. Fish species sampled in headwater streams of the rio Machado basin, with their abundance (N) and the catalogue number of the voucher specimens. Classification follows Reis et al. (2003); except for Serrasalmidae that follows Calcagnotto et al. (2005) and Parauchenipterus porosus that follows Buckup et al. (2007). Single quotation marks indicate provisional genera and asterisk indicate non-native species.

\begin{tabular}{|c|c|c|}
\hline TAXON & $\mathbf{N}$ & VOUCHER \\
\hline \multicolumn{3}{|l|}{ MYLIOBATIFORMES } \\
\hline \multicolumn{3}{|l|}{ Potamotrygonidae } \\
\hline Potamotrygon orbignyi (Castelnau, 1855) & 1 & DZSJRP 17112 \\
\hline \multicolumn{3}{|l|}{ CHARACIFORMES } \\
\hline \multicolumn{3}{|l|}{ Parodontidae } \\
\hline Parodon nasus Kner, 1859 & 4 & DZSJRP 14506 \\
\hline \multicolumn{3}{|l|}{ Curimatidae } \\
\hline Curimatopsis macrolepis (Steindachner, 1876) & 6 & DZSJRP 16692 \\
\hline Cyphocharax plumbeus (Eigenmann and Eigenmann, 1889) & 1 & DZSJRP 17238 \\
\hline Cyphocharax spiluropsis (Eigenmann and Eigenmann, 1889) & 40 & DZSJRP 16630 \\
\hline Steindachnerina cf. dobula (Günther, 1868) & 4 & DZSJRP 14512 \\
\hline Steindachnerina fasciata (Vari and Géry, 1985) & 57 & DZSJRP 14661 \\
\hline Steindachnerina guentheri (Eigenmann and Eigenmann, 1889) & 3 & DZSJRP 16782 \\
\hline \multicolumn{3}{|l|}{ Prochilodontidae } \\
\hline Prochilodus nigricans Spix and Agassiz, 1829 & 1 & DZSJRP 16799 \\
\hline \multicolumn{3}{|l|}{ Anostomidae } \\
\hline Anostomus ternetzi Fernández-Yépez, 1949 & 5 & DZSJRP 14664 \\
\hline Leporinus friderici (Block, 1794) & 36 & DZSJRP 14763 \\
\hline \multicolumn{3}{|l|}{ Crenuchidae } \\
\hline Characidium aff. gomesi Travassos, 1956 & 7 & DZSJRP 14704 \\
\hline Characidium aff. zebra Eigenmann, 1909 & 762 & DZSJRP 14703 \\
\hline Characidium sp. & 8 & DZSJRP 14335 \\
\hline Elachocharax pulcher Myers, 1927 & 79 & DZSJRP 15057 \\
\hline Microcharacidium sp. & 50 & DZSJRP 16653 \\
\hline Microcharacidium aff. weitzmani Buckup, 1996 & 38 & DZSJRP 14986 \\
\hline Melanocharacidium dispilomma Buckup, 1993 & 1 & DZSJRP 17205 \\
\hline Melanocharacidium pectorale Buckup, 1993 & 1 & DZSJRP 16678 \\
\hline \multicolumn{3}{|l|}{ Hemiodontidae } \\
\hline Hemiodus unimaculatus (Block, 1794) & 2 & DZSJRP 14672 \\
\hline Gasteropelecidae & & \\
\hline
\end{tabular}


TABLE 2. Continued.

\begin{tabular}{|c|c|c|}
\hline TAXON & $\mathbf{N}$ & VOUCHER \\
\hline Carnegiella strigata (Günther, 1864) & 40 & DZSJRP 14886 \\
\hline \multicolumn{3}{|l|}{ Characidae } \\
\hline Amazonspinther dalmata Bührnheim, Carvalho, Malabarba and Weitzman, 2008 & 7 & DZSJRP 14947 \\
\hline Astyanax cf. bimaculatus (Linnaeus, 1758) & 108 & DZSJRP 14419 \\
\hline Astyanax cf. maximus (Steindachner, 1876) & 18 & DZSJRP 14460 \\
\hline Astyanax maculisquamis Garutti and Britski, 1997 & 43 & DZSJRP 14700 \\
\hline Bario steindachneri (Eigenmann, 1893) & 3 & DZSJRP 15090 \\
\hline Brachychalcinus copei (Steindachner, 1822) & 147 & DZSJRP 14769 \\
\hline Bryconops caudomaculatus (Günther, 1864) & 912 & DZSJRP 14628 \\
\hline Bryconops piracolina Wingert and Malabarba, 2011 & 23 & DZSJRP 17278 \\
\hline Bryconella pallidifrons (Fowler, 1946) & 695 & DZSJRP 16651 \\
\hline 'Cheirodon' troemneri Fowler, 1942 & 62 & DZSJRP 14668 \\
\hline Creagrutus petilus Vari and Harold, 2001 & 1021 & DZSJRP 14733 \\
\hline Hemigrammus sp. & 14 & DZSJRP 15101 \\
\hline Hemigrammus aff. ocellifer (Steindachner, 1882) & 62 & DZSJRP 15009 \\
\hline Hemigrammus bellotti (Steindachner, 1882) & 152 & DZSJRP 14524 \\
\hline Hemigrammus melanochrous Fowler, 1913 & 1418 & DZSJRP 15100 \\
\hline Hemigrammus neptunus Zarske and Géry, 2002 & 60 & DZSJRP 14710 \\
\hline Hyphessobrycon aff. heterorhabdus (Ulrey, 1894) & 144 & DZSJRP 16929 \\
\hline Hyphessobrycon agulha Fowler, 1913 & 1131 & DZSJRP 15103 \\
\hline Hyphessobrycon bentosi Durbin, 1908 & 178 & DZSJRP 15011 \\
\hline Hyphessobrycon copelandi Durbin, 1908 & 151 & DZSJRP 14673 \\
\hline Jupiaba citrina Zanata and Ohara, 2009 & 273 & DZSJRP 14701 \\
\hline Jupiaba poranga Zanata, 1997 & 9 & DZSJRP 15107 \\
\hline Jupiaba zonata (Eigenmann, 1908) & 55 & DZSJRP 19916 \\
\hline Knodus cf. smithi Fowler, 1913 & 827 & DZSJRP 14715 \\
\hline Knodus heteresthes Eigenmann, 1908 & 736 & DZSJRP 14651 \\
\hline Microschemobrycon guaporensis Eigenmann, 1915 & 166 & DZSJRP 14476 \\
\hline Moenkhausia aff. gracilima Eigenmann, 1908 & 1 & DZSJRP 16817 \\
\hline Moenkhausia cf. bonita Benine, Castro and Sabino, 2004 & 339 & DZSJRP 14717 \\
\hline Moenkhausia cf. justae Eigenmann, 1908 & 41 & DZSJRP 14526 \\
\hline Moenkhausia collettii (Steindachner, 1882) & 1924 & DZSJRP 14639 \\
\hline Moenkhausia cotinho Eigenmann, 1908 & 259 & DZSJRP 14478 \\
\hline Moenkhausia grandisquamis Müller and Troschel, 1845 & 11 & DZSJRP 14962 \\
\hline Moenkhausia mikia Marinho and Langeani, 2010 & 105 & DZSJRP 14447 \\
\hline Moenkhausia oligolepis (Günther, 1864) & 330 & DZSJRP 14479 \\
\hline Moenkhausia pirauba Zanata, Birindelli and Moreira, 2010 & 19 & DZSJRP 15112 \\
\hline Odontostilbe fugitiva Cope, 1870 & 307 & DZSJRP 14545 \\
\hline Phenacogaster retropinnus Lucena and Malabarba, 2010 & 386 & DZSJRP 14450 \\
\hline Serrapinnus aff. notomelas (Eigenmann, 1915) & 3642 & DZSJRP 14659 \\
\hline Serrapinnus microdon (Eigenmann, 1915) & 1901 & DZSJRP 14658 \\
\hline Tetragonopterus argenteus Cuvier, 1816 & 2 & DZSJRP 17040 \\
\hline Triportheus angulatus (Spix and Agassiz, 1829) & 2 & DZSJRP 14456 \\
\hline Tyttocharax madeirae Fowler, 1913 & 32 & DZSJRP 14945 \\
\hline \multicolumn{3}{|l|}{ Serrasalmidae } \\
\hline Myleus sp. & 12 & DZSJRP 14741 \\
\hline Serrasalmus rhombeus (Linnaeus, 1766) & 1 & DZSJRP 14695 \\
\hline \multicolumn{3}{|l|}{ Acestrorhynchidae } \\
\hline Acestrorhynchus falcatus (Bloch, 1794) & 3 & DZSJRP 17072 \\
\hline \multicolumn{3}{|l|}{ Erythrinidae } \\
\hline Erythrinus erythrinus (Bloch and Schneider, 1801) & 11 & DZSJRP 16650 \\
\hline Hoplerythrinus unitaeniatus (Spix and Agassiz, 1829) & 3 & DZSJRP 16764 \\
\hline Hoplias malabaricus (Bloch, 1794) & 88 & DZSJRP 14538 \\
\hline \multicolumn{3}{|l|}{ Lebiasinidae } \\
\hline Nannostomus trifasciatus Steindachner, 1876 & 1 & DZSJRP 14963 \\
\hline Pyrrhulina cf. australis Eigenmann and Kennedy, 1903 & 193 & DZSJRP 14634 \\
\hline Pyrrhulina cf. brevis Steindachner, 1876 & 65 & DZSJRP 15115 \\
\hline Pyrrhulina cf. zigzag Zarske and Géry, 1997 & 9 & DZSJRP 17280 \\
\hline \multicolumn{3}{|l|}{ SILURIFORMES } \\
\hline \multicolumn{3}{|l|}{ Cetopsidae } \\
\hline Denticetopsis seducta (Vari, Ferraris and de Pinna, 2005) & 4 & DZSJRP 14887 \\
\hline Helogenes gouldingi Vari and Ortega, 1986 & 22 & DZSJRP 15099 \\
\hline
\end{tabular}


TABle 2. Continued.

\begin{tabular}{|c|c|c|}
\hline TAXON & $\mathbf{N}$ & VOUCHER \\
\hline \multicolumn{3}{|l|}{ Aspredinidae } \\
\hline Pseudobunocephalus amazonicus (Mees, 1989) & 37 & DZSJRP 14940 \\
\hline \multicolumn{3}{|l|}{ Trichomycteridae } \\
\hline Ituglanis amazonicus (Steindachner, 1882) & 108 & DZSJRP 14676 \\
\hline Miuroglanis platycephalus Eigenmann and Eigenmann, 1889 & 1 & DZSJRP 14963 \\
\hline Paracanthopoma sp. & 19 & DZSJRP 14905 \\
\hline \multicolumn{3}{|l|}{ Callichthyidae } \\
\hline Corydoras acutus Cope, 1872 & 5 & DZSJRP 15023 \\
\hline Corydoras aff. ambiacus Cope, 1872 & 3 & DZSJRP 17229 \\
\hline Corydoras cf. melanistius Regan, 1912 & 55 & DZSJRP 15124 \\
\hline Corydoras bondi Gosline, 1940 & 1 & DZSJRP 17263 \\
\hline Corydoras elegans Steindachner, 1876 & 7 & DZSJRP 14422 \\
\hline Corydoras stenocephalus Eigenmann and Allen, 1942 & 5 & DZSJRP 16757 \\
\hline Corydoras trilineatus Cope, 1872 & 82 & DZSJRP 14755 \\
\hline Hoplosternum littorale (Hancock, 1828) & 7 & DZSJRP 14423 \\
\hline Megalechis picta (Müller and Troschel, 1849) & 49 & DZSJRP 16753 \\
\hline \multicolumn{3}{|l|}{ Loricariidae } \\
\hline Ancistrus lithurgicus Eigenmann, 1912 & 290 & DZSJRP 14418 \\
\hline Farlowella cf. oxyrryncha (Kner, 1853) & 120 & DZSJRP 14671 \\
\hline Squaliforma emarginata (Valenciennes, 1840) & 22 & DZSJRP 14712 \\
\hline Hypostomus sp. & 1 & DZSJRP 17290 \\
\hline Hypostomus pyrineusi (Miranda Ribeiro, 1920) & 34 & DZSJRP 14424 \\
\hline Lasiancistrus schomburgkii (Günther, 1864) & 61 & DZSJRP 14697 \\
\hline Loricaria cataphracta Linnaeus, 1758 & 4 & DZSJRP 14499 \\
\hline Otocinclus hoppei Miranda Ribeiro, 1939 & 119 & DZSJRP 14685 \\
\hline Parotocinclus aff. aripuanensis Garavello, 1988 & 24 & DZSJRP 14895 \\
\hline Rineloricaria sp. & 6 & DZSJRP 14635 \\
\hline Rineloricaria heteroptera Isbrücker and Nijssen, 1976 & 164 & DZSJRP 14427 \\
\hline Spatuloricaria evansii (Boulenger, 1892) & 4 & DZSJRP 14511 \\
\hline \multicolumn{3}{|l|}{ Pseudopimelodidae } \\
\hline Batrochoglanis cf. raninus (Valenciennes, 1840) & 16 & DZSJRP 14969 \\
\hline Batrochoglanis villosus (Eigenmann, 1912) & 5 & DZSJRP 14665 \\
\hline Microglanis poecilus Eigenmann, 1912 & 1 & DZSJRP 16655 \\
\hline \multicolumn{3}{|l|}{ Heptapteridae } \\
\hline Cetopsorhamdia sp. 1 & 24 & DZSJRP 17295 \\
\hline Cetopsorhamdia sp. 2 & 8 & DZSJRP 17279 \\
\hline Cetopsorhamdia sp. 3 & 6 & DZSJRP 17216 \\
\hline Imparfinis cf. hasemani Steindachner, 1917 & 124 & DZSJRP 14714 \\
\hline Imparfinis stictonotus (Fowler, 1940) & 49 & DZSJRP 14471 \\
\hline Phenacorhamdia cf. boliviana (Pearson, 1924) & 4 & DZSJRP 14688 \\
\hline Phenacorhamdia sp. & 70 & DZSJRP 15019 \\
\hline Pimelodella sp. & 11 & DZSJRP 14527 \\
\hline Pimelodella cf. howesi Fowler, 1940 & 55 & DZSJRP 14656 \\
\hline Rhamdia quelen (Quoy and Gaimard, 1824) & 6 & DZSJRP 14770 \\
\hline \multicolumn{3}{|l|}{ Doradidae } \\
\hline Acanthodoras cataphractus (Linnaeus, 1758) & 19 & DZSJRP 16687 \\
\hline \multicolumn{3}{|l|}{ Auchenipteridae } \\
\hline Centromochlus cf. perugiae Steindachner, 1882 & 1 & DZSJRP 17261 \\
\hline Tatia aulopygia (Kner, 1858) & 2 & DZSJRP 14696 \\
\hline Parauchenipterus porosus (Eigenmann and Eigenmann, 1888) & 5 & DZSJRP 17038 \\
\hline \multicolumn{3}{|l|}{ GYMNOTIFORMES } \\
\hline \multicolumn{3}{|l|}{ Gymnotidae } \\
\hline Gymnotus aff. arapaima Albert and Crampton, 2001 & 26 & DZSJRP 14649 \\
\hline Gymnotus carapo Linnaeus, 1758 & 35 & DZSJRP 14648 \\
\hline Gymnotus coropinae Hoederman, 1962 & 81 & DZSJRP 15006 \\
\hline \multicolumn{3}{|l|}{ Sternopygidae } \\
\hline Eigenmannia trilineata López and Castello, 1966 & 196 & DZSJRP 14406 \\
\hline Sternopygus macrurus (Bloch and Schneider, 1801) & 99 & DZSJRP 14484 \\
\hline \multicolumn{3}{|l|}{ Rhamphichthyidae } \\
\hline Gymnorhamphichthys petiti Géry and Vu-Tân-Tuê, 1964 & 287 & DZSJRP 14631 \\
\hline \multicolumn{3}{|l|}{ Hypopomidae } \\
\hline Brachyhypopomus sp. 1 & 2 & DZSJRP 14627 \\
\hline
\end{tabular}


TABLE 2. CONTINUED.

\begin{tabular}{|c|c|c|}
\hline TAXON & $\mathbf{N}$ & VOUCHER \\
\hline Brachyhyроротиs sp. 2 & 15 & DZSJRP 15091 \\
\hline Brachyhyроротиs sp. 3 & 26 & DZSJRP 15092 \\
\hline Hypopygus lepturus Hoedeman, 1962 & 128 & DZSJRP 14632 \\
\hline \multicolumn{3}{|l|}{ Apteronotidae } \\
\hline Apteronotus albifrons (Linnaeus, 1766) & 6 & DZSJRP 14641 \\
\hline Platyurosternarchus macrostomus (Günter, 1864) & 2 & DZSJRP 14690 \\
\hline \multicolumn{3}{|l|}{ CYPRINODONTIFORMES } \\
\hline \multicolumn{3}{|l|}{ Rivulidae } \\
\hline Rivulus sp. & 4 & DZSJRP 14942 \\
\hline \multicolumn{3}{|l|}{ BELONIFORMES } \\
\hline \multicolumn{3}{|l|}{ Belonidae } \\
\hline Potamorrhaphis eigenmanni Miranda Ribeiro, 1915 & 2 & DZSJRP 14949 \\
\hline \multicolumn{3}{|l|}{ SYNBRANCHIFORMES } \\
\hline \multicolumn{3}{|l|}{ Synbranchidae } \\
\hline Synbranchus marmoratus Bloch, 1795 & 22 & DZSJRP 14485 \\
\hline \multicolumn{3}{|l|}{ PERCIFORMES } \\
\hline \multicolumn{3}{|l|}{ Cichlidae } \\
\hline Aequidens tetramerus (Heckel, 1840) & 199 & DZSJRP 14626 \\
\hline Apistogramma cf. resticulosa Kullander, 1980 & 563 & DZSJRP 14994 \\
\hline Cichlasoma amazonarum Kullander, 1983 & 46 & DZSJRP 14462 \\
\hline Crenicichla johanna Heckel, 1840 & 2 & DZSJRP 14758 \\
\hline Crenicichla santosi Ploeg, 1991 & 163 & DZSJRP 14757 \\
\hline Geophagus megasema Heckel, 1840 & 1 & DZSJRP 15004 \\
\hline Satanoperca jurupari (Heckel, 1840) & 60 & DZSJRP 14636 \\
\hline Tilapia rendalli (Boulenguer, 1897) * & 2 & DZSJRP 14431 \\
\hline TOTAL & 22875 & \\
\hline
\end{tabular}

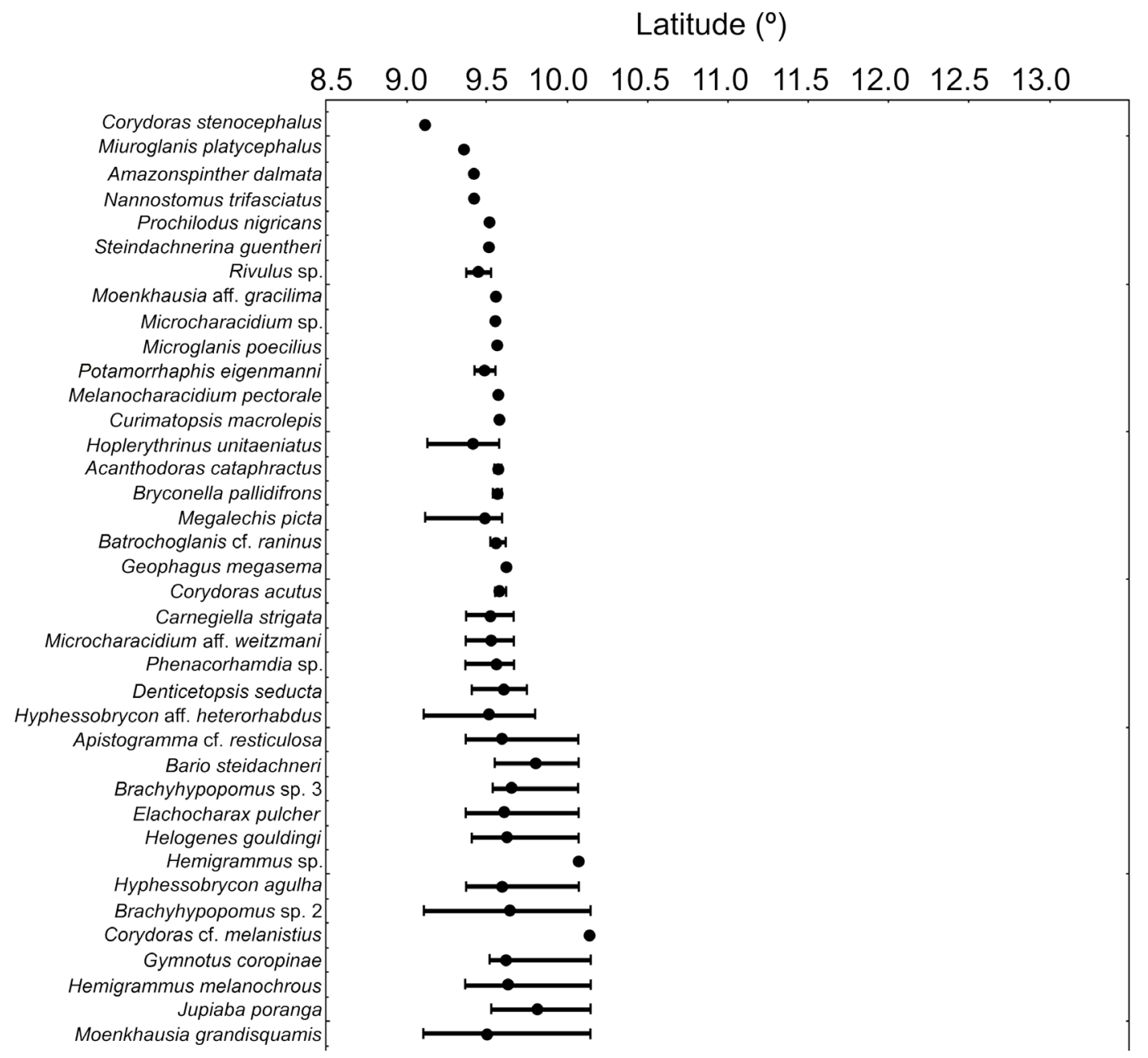

FIGURE 3. Latitudinal distribution of 140 species collected in the rio Machado basin. Bars indicate latitudinal range for each species, dots indicate average latitude. 


\section{Latitude $\left(^{\circ}\right)$}

\section{$\begin{array}{llllllllll}8.5 & 9.0 & 9.5 & 10.0 & 10.5 & 11.0 & 11.5 & 12.0 & 12.5 & 13.0\end{array}$}

Pseudobunocephalus amazonicus

Pyrrhulina cf. brevis

Hemigrammus bellotti Steindachnerina cf. dobula

Jupiaba zonata

Loricaria cataphracta

Crenicichla johanna

Potamotrygon orbigny

Tilapia rendalli

Corydoras elegan

Tetragonopterus argenteus

Hoplosternum littorale

Brachyhypopomus sp.

Rineloricaria sp.

Parauchenipterus porosus

Corydoras aff. ambiacus

Cyphocharax plumbeus

Hypopygus lepturus

Odontostilbe fugitiva

Triportheus angulatus

Cetopsorhamdia sp.

Melanocharacidium dispilomma

Anostomus ternetzi

Batrochoglanis villosus Characidium $\mathrm{sp}$.

'Cheirodon' troemner

Hemiodus unimaculatus

Hyphessobrycon copeland Parodon nasus

Platyurosternachus macrostomus

Serrasalmus rhombeus

Tatia aulopygia

Apteronotus albifrons

Hemigrammus aff. ocellifer Microschemobrycon guaporensis

Moenkhausia mikia

Pyrrhulina cf. australis Phenacorhamdia cf. bolivian

Acestrorhynchus falcatus

Moenkhausia pirauba

Synbranchus marmoratus

Paracanthopoma sp.

Rhamdia quelen

Cyphocharax spiluropsis

Gymnotus aff. arapaima

Hyphessobrycon bentosi

Lasiancistrus schomburgki

Leporinus friderici

Astyanax maculisquamis

Characidium aff. gomesi

Cichlasoma amazonarum

Gymnotus carapo

Hemigrammus neptunus

Hoplias malabaricus

Squaliforma emarginata

Imparfinis cf. hasemani

Moenkhausia cf. bonita

Moenkhausia cf. justae

Moenkhausia cotinho

Satanoperca jurupari

Spatuloricaria evansi

Astyanax cf. maximus

Imparfinis stictonotus

Knodus heteresthes

Otocinclus hoppe

Pimelodella sp.

Tyttocharax madeirae

Ancistrus lithurgicus

Brachychalcinus cope

Corydoras trilineatus

Ituglanis amazonicus Myleus sp.

Phenacogaster retropinnus

Rineloricaria heteroptera

Serrapinnus microdon

Serrapinnus aff. notomelas

Bryconops piracolina

Pyrrhulina cf. zigzag

Cetopsorhamdia sp. 2

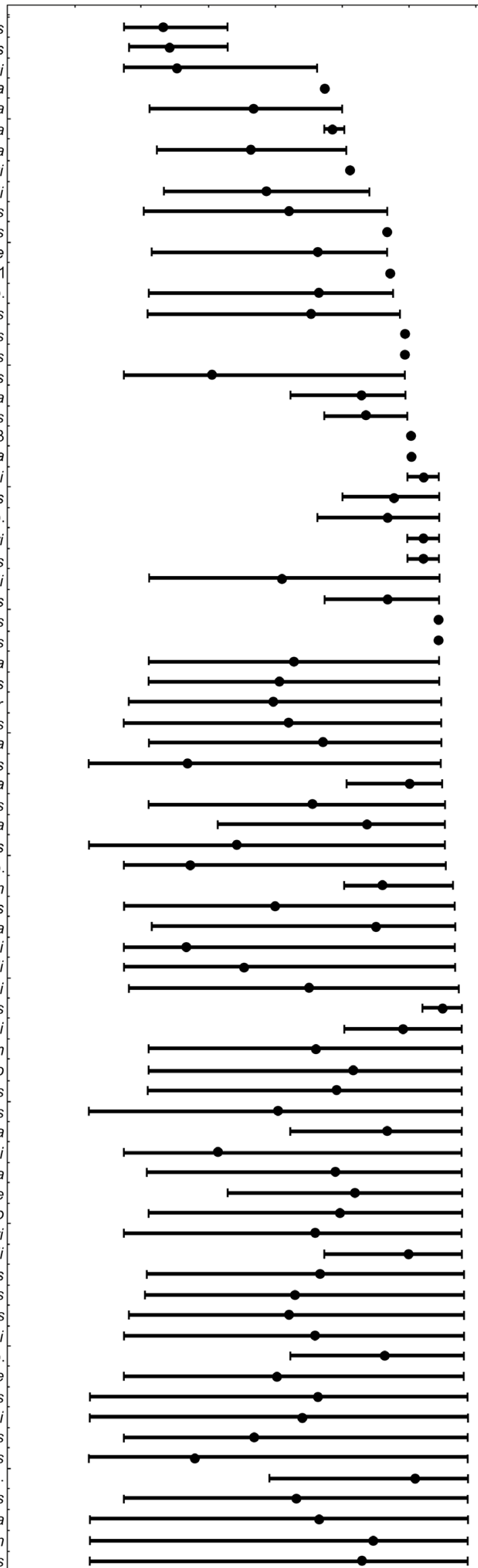


Latitude $\left({ }^{\circ}\right)$

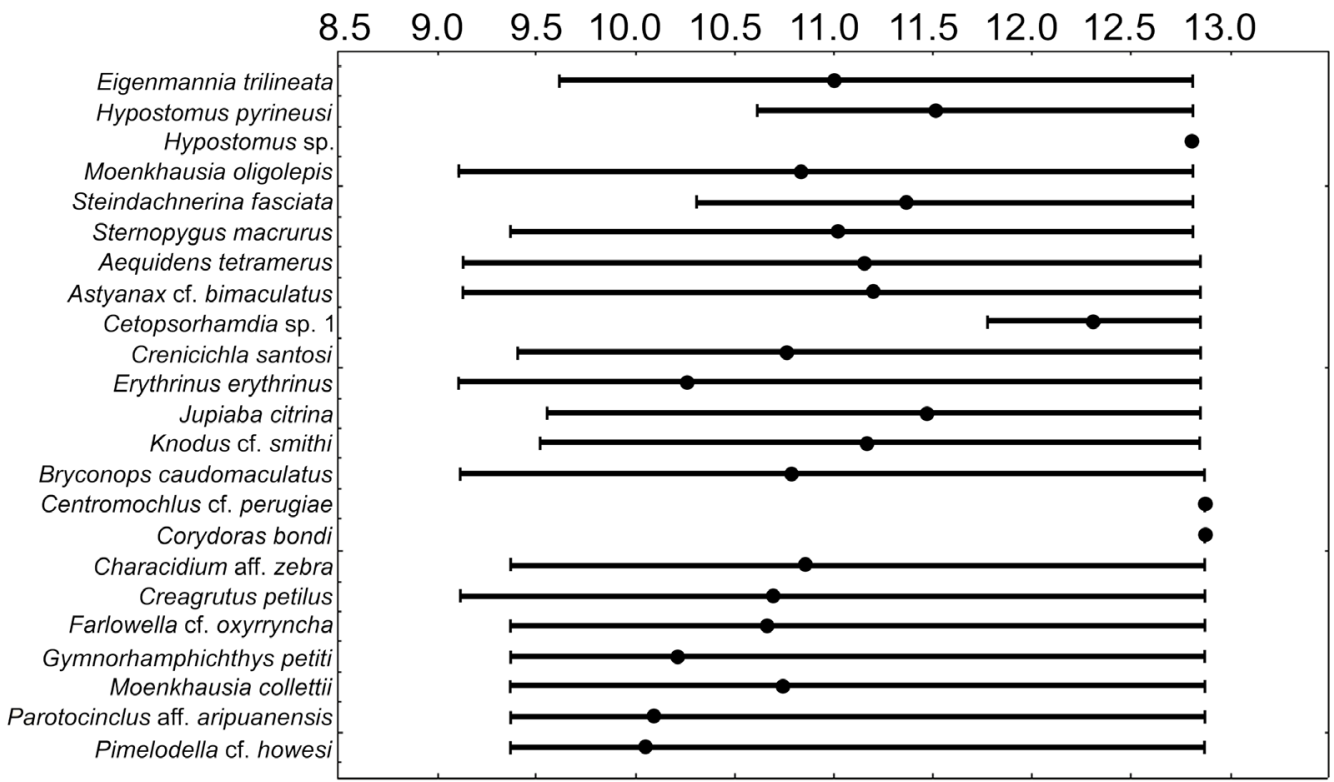

Figure 3. CONTINUED.

ACKNowledgments: We are grateful to Bárbara Callegari (MCP/ PUCRS), Ilana Fichberg (MZUSP), Fernanda Martins (UNESP), Flávio Lima (UNICAMP), Francisco Langeani (UNESP), Leandro Sousa (UFPA), Manoela Marinho (MZUSP), Marcelo Britto (MNRJ), Marcelo Carvalho (IB/USP), and William Ohara (MZUSP) for help with fish identifications; to members of the ichthyology laboratories from UNESP and UNIR for help with the field work; to ICMBio (REBio Jaru) for logistical support; to SEDAM and to the extractivists' associations for permission to carry out the surveys in the areas of RESEX Rio Preto-Jacundá, RESEX Castanheira and RESEX Aquariquara; to "Fundação de Amparo à Pesquisa do Estado de São Paulo" for financial support (2010/17494-8) and fellowships to FRC (2011/11422-8) and GLB (2011/11677-6). MAPM receives fellowship from "Programa de Apoio a Estudantes de Doutorado do Exterior" (AUIP/UNESP) and LC is granted by "Conselho Nacional de desenvolvimento Científico e Tecnológico” (306758/2010-5).

\section{LITERATURE CITED}

ANA (Agência Nacional de Águas). 2013. Database accessible at http:// hidroweb.ana.gov.br/. Captured on October 2013.

Buckup, P.A., N.A Menezes, M.S. Ghazzi (ed.). 2007. Catálogo das espécies de peixes de água doce do Brasil. Rio de Janeiro: Série Livros 23. 195 p.

Barros, D.F., J. Zuanon, F.P. de Mendonça, H.M.V. Espírito-Santo, A.V. Galuch and A.L.M. Albernaz. 2011. The fish fauna of streams in the MadeiraPurus interfluvial region, Brazilian Amazon. Check List 7(1): 768-773.

Calcagnotto, D., S.A. Schaefer and R. DeSalle. 2005. Relationships among characiform fishes inferred from analysis of nuclear and mitochondrial gene sequences. Molecular Phylogenetics and Evolution 36(1): 135-153.

Camargo, M. and T. Giarrizzo. 2007. Fish, Marmelos Conservation Area (BX044), Madeira River basin, states of Amazonas and Rondônia, Brazil. Check List 3(4): 291-296.

Castro, R.M.C. 1999. Evolução da ictiofauna de riachos sul-americanos: padrões gerais e possíveis processos causais; p. 139-155 In E.P. Caramaschi, R. Mazzoni, C.R.S.F. Bizerril, P.R. and Peres-Neto (ed.). Ecologia de Peixes de Riachos: Estado Atual e Perspectivas. Oecologia Brasiliensis. Volume VI. Rio de Janeiro: PPGE-UFRJ.

Castro, R.M.C. and N.A. Menezes. 1998. Estudo diagnóstico da diversidade de peixes do Estado de São Paulo; p. 1-13 In R.M.C. Castro (ed.) Biodiversidade do Estado de São Paulo, Brasil: Síntese do conhecimento ao final do século XX. São Paulo: WinnerGraph.

Castro, R.M.C., L. Casatti, H.F. Santos, K.M. Ferreira, A.C. Ribeiro, R.C Benine, G.Z.P. Dardis, A.L.A. Melo, T.X. Abreu, F.A. Bockmann, M. Carvalho, F.Z. Gibran and F.C.T. Lima. 2003. Estrutura e composição da ictiofauna de riachos do Rio Paranapanema, sudeste e sul do Brasil. Biota Neotropica 3(1): 1-31.

Cowell, R.K. 2005. EstimateS 7.5.2. Statistical estimation of species richness and shared species from samples. Software. University of Connecticut.

Colwell, R.K., C.X. Mao and J. Chang. 2004. Interpolating, extrapolating, and comparing incidence-based species accumulation curves. Ecology 85: 2717-2727.
Dale, V.H., R.V.O' Neill, M. Pedlowski and F. Southworth. 1993. Causes and effects of land-use change in central Rondônia, Brazil. Photogrammetric Engineering \& Remote Sensing 59(6): 997-1005.

Fernandes, L.C. and S.C.P. Guimarães. 2002. Atlas geoambiental de Rondônia. Porto Velho: SEDAM. 141 p.

Ferraz, S.F.B., C.A. Vettorazzi and D.M. Theobald. 2009. Using indicators of deforestation and land-use dynamics to support conservation strategies: a case study of central Rondônia, Brazil. Forest Ecology and Management 257(7): 1586-1595.

INPE (Instituto Nacional de Pesquisas Espaciais). 2010. Projeto Prodes Monitoramento da Floresta Amazônica Brasileira por Satélite. Electronic Database accessible at http://www.obt.inpe.br/prodes/ index.html. Captured on October 2010.

Krusche, A.V., M.V.R. Ballester, R.L. Victoria, M.C. Bernardes, N.K. Leite, L. Hanada, D.C. Victoria, A.M. Toledo, J.P. Ometto, M.Z. Moreira, B.M. Gomes, M.A. Bolson, S. Gouveia Neto, N. Bonelli, L. Deegan, C. Neill, S. Thomas, A.K. Aufdenkampe and J.E. Richey. 2005. Efeitos das mudanças do uso da terra na biogeoquímica dos corpos d'água da bacia do Rio Ji-Paraná, Rondônia. Acta Amazonica 35(2): 197-205.

Lee, S.M. and A. Chao. 1994. Estimating population size via sample coverage for closed capture-recapture models. Biometrics 50(1): 8897.

Lowe-McConnell, R.H. 1999. Estudos ecológicos de comunidades de peixes tropicais. São Paulo: Editora da Universidade de São Paulo. 534 p.

Martins, D.P. 2008. Novos caminhos e antigas práticas: acordos de comunidades com empresas para o manejo florestal, o caso da Reserva Extrativista Rio Preto Jacundá em Machadinho D'Oeste-RO. Belém: UFPA. 182 p.

Moreira, R.C.S., C.A.S. Müller and O. Siena. 2010. Análise da viabilidade econômica da Reserva Extrativista Rio Preto Jacundá sob o enfoque da renda média nominal mensal da população tradicional. Campo Grande: Sociedade Brasileira de Economia (SOBER), Administração e Sociologia Rural. 16 p.

Perin, L., O.A. Shibatta and P.S. Bernarde. 2007. Fish, Machado River basin, Cacoal urban area, state of Rondônia, Brazil. Check List 3(2): 94-97.

Reis, R.E., S.O. Kullander and C.J. Ferraris (ed.). 2003. Check List of the Freshwater Fishes of South and Central America. Porto Alegre: Edipucrs. 729 p.

SpeciesLink. 2013. The SpeciesLink network. Electronic Database accessible at http://www.splink.org.br. Captured on March 2013.

Strahler, A.N. 1957. Quantitative analysis of watershed geomorphology. Transaction of American Geophysical Union 38: 913-920.

RECEIVED: March 2013

ACCEPTED: November 2013

Published ONLINE: November 2013

EDITORIAL RESPONSIBILITY: Tiago Pinto Carvalho 